\title{
Leader-Following Bounded Consensus and Multiconsensus of Multiagent Systems
}

\author{
Chao Long, ${ }^{1}$ Chunxi Yang $\mathbb{D},{ }^{1}$ Guangsong Han, ${ }^{2}$ Yingbo Huang, ${ }^{1}$ and Shichang Han ${ }^{1}$ \\ ${ }^{1}$ Faculty of Mechanical and Electrical Engineering, Kunming University of Science and Technology, Kunming 650500, China \\ ${ }^{2}$ Joint Operations College, PLA National Defense University, Shijiazhuang 050084, China \\ Correspondence should be addressed to Chunxi Yang; ycx2003@163.com
}

Received 18 August 2020; Revised 11 September 2020; Accepted 14 September 2020; Published 13 October 2020

Academic Editor: Shubo Wang

Copyright (c) 2020 Chao Long et al. This is an open access article distributed under the Creative Commons Attribution License, which permits unrestricted use, distribution, and reproduction in any medium, provided the original work is properly cited.

This paper investigates the problem of leader-following consensus and multiconsensus of multiagent systems. A leader-following and bounded consensus protocol via impulsive control for multiagent systems is proposed by using sampled position and velocity data. Consensus and multiconsensus commutative evolution stimulated by varying intelligence degrees of each agent can be achieved to avoid obstacles. Especially, the multiconsensus leader-following can be obtained without grouping the multiagent networks in advance. The necessary and sufficient condition is given to the leader-following bounded consensus tracking of the system by using the Hurwitz criterion and properties of the Laplacian matrix. A simulation is provided to verify the availability of the proposed impulsive control protocol. Furthermore, the result can be applied in obstacle avoidance and round up of target by regulating the intelligence degrees.

\section{Introduction}

In recent years, multiagent systems have attracted a great deal of attention from researchers because agents can communicate and coordinate with each other for achieving various complex tasks, such as wireless sensor navigation [1], distributed strikes of missiles [2], and coordination of robots [3-7]. Olfati-saber Murray [8] proposed the concept of consensus protocol for multiagent systems, and the consensus of multiagent systems with directed topology, undirected topology, and switching topology was studied. Ren and Beard [9] studied the directed switching topology, and their results showed that if the network topology contains a directed spanning tree, then the multiagent systems will achieve consensus. At present, tons of research studies have been conducted about the consensus of multiagent systems, such as the delay [10], heterogeneous [11], and aperiodic sampled [12] studies.

As a special case of consensus, multiconsensus/group consensus has received much attention for its multiconvergent states and promising potential applications.
$\mathrm{Pu}$ et al. [13] investigated the group consensus for discretetime heterogeneous multiagent systems with input time delays. Ji et al. [14] investigated group consensus based on linearly coupled multiagent networks, and they extended their work to study the group consensus for the multiagent network with generally connected topology which neither needs to be strongly connected nor needs to contain a directed spanning tree. Shang [15] studied the L-1 group consensus problem of discrete-time multiagent systems with external stochastic inputs. L-1 group consensus criteria were obtained for multiagent systems with switching topologies. Furthermore, a large number of results were produced, such as multiconsensus of continuous-time systems [16], tracking control [17], and event-driver control [18]. Considering that most of the studies were performed by presenting groupings, Han et al. [19] proposed the concept of intelligence degree and also gave necessary and sufficient conditions of multiconsensus with fixed intelligence degree. By adjusting the intelligent degree of each agent, it is possible to regulate the dynamic behaviour of the multiagent system. 
All agents following the trajectory of the leader is called leader-following consensus in multiagent systems. Leaderfollowing consensus is one of the hot topics of multiagent systems, which are widely applied in military field, such as striking a known dynamic target [20] and UAV formation [21]. Considering a nonlinear multiagent system, $\mathrm{He}$ et al. [22] proposed a distributed consensus protocol with probabilistic sampling in two sampling periods and solved the problem of sampled-data leader-following consensus of a group of agents with nonlinear characteristics. Zhu et al. [23] achieved the leader-following consensus by adaptive event control. Ren et al. [24] designed a fuzzy adaptive control mechanism for nonlinear multiagent systems with unknown control directions. However, as far as I am concerned, almost all works mentioned above paid attention to how to obtain a consensus protocol or better consensus performances under the given communication constraints. Currently, some works discussed how to avoid static obstacles and dynamic obstacles during the process of following the trajectory of the leader under given environment constraints. Douthwaite et al. [25] presented a critical analysis of some of the most promising approaches to geometric collision avoidance in multiagent systems, and the respective features and limitations of each algorithm were discussed and presented through examples. Guo et al. [26] used model predictive control to realize collision avoidance of multiagent systems. This technique has strong practical significance especially in dynamic path planning, round up, and battle among USV or UAV. On the one hand, the leader tries to plan an available path in order to overcome static obstacles [27] and dynamic obstacles [28]. On the other hand, all group members should build an effective mechanism to avoid obstacles when the preplanned path is disrupted by moving obstacles.

Therefore, combined with leader-following mechanism, obstacle avoidance, and consensus of multiagent systems, the problem of leader-following consensus and multiconsensus of second-order continuous-time multiagent systems is considered in this paper. A leader-following bounded consensus protocol with different intelligence degrees via impulsive control is proposed. All grouped members follow the trajectory of the virtual leader in the dynamic process, and then, dynamic obstacles which hinder the preplanned path are avoided by regulating suitable intelligence degree of agents which results in varying grouping and different dynamic behaviours of the system. Sufficient and necessary conditions of multiconsensus leader-following of multiagent systems via impulsive control are given. Some simulation results show that the impulsive control protocol with different intelligent degrees can realize the consensus and multiconsensus leader-following.

The rest of paper is organized as follows. In Section 2, we provide some preliminary notions including lemmas in graph theory and matrix theory. The impulsive consensus leader-following protocol is also introduced, and the main result of this paper, that is, the bounded convergence of the proposed protocol, is presented in Section 3. In Section 4, an illustrative numerical example is given. The paper is concluded in Section 5.

\section{Preliminaries and Problem Formulation}

2.1. Notions from Graph Theory. Let $G=(V, E, A)$ be a digraph with the set of nodes $V=\{1, \ldots, n\}$, the set of edges $E \subseteq V \times V$, and the weighted adjacency matrix $A=\left[a_{i j}\right] \in R^{n \times n}$ in which $a_{i j} \neq 0$ if $(j, i) \in E$ and $a_{i j}=0$ otherwise. Then, weight reuse $d_{i}=\sum_{j=1}^{n} a_{i j}$ for node $i$, and the diagonal matrix $D=\operatorname{diag}\left(d_{1}, \ldots, d_{n}\right)$ represents the input weight matrix of the directed graph $G$.

The Laplace matrix $L=\left[l_{i j}\right] \in R^{n \times n}$ of $G$ is defined as

$$
l_{i j}= \begin{cases}-a_{i j}, & j \neq i, \\ \sum_{k=1, k \neq i}^{n} a_{i k}, & j=i .\end{cases}
$$

A directed path is a sequence of edges in a digraph of the form $\left(i_{1}, i_{2}\right) \ldots\left(i_{n-1}, i_{n}\right)$ with all distinct $i_{j}$. A directed tree is a digraph, where there exists an agent, called the root, such that any other agent of the digraph can be reached by one and only one path starting at the root [29].

2.2. Preliminaries. Assume that agent $i$ evolves according to the multiagent system dynamics:

$$
\left\{\begin{array}{l}
\dot{x}_{i}(t)=v_{i}(t), \\
\dot{v}_{i}(t)=u_{i}(t), \quad \forall i \in V,
\end{array}\right.
$$

where $x_{i}(t)$ and $v_{i}(t) \in R^{n}$ are the position and velocity states of the agent $i$, respectively, and $u_{i}(t) \in R^{n}$ is its control input. The communications between agents are given by a digraph $G=\{V, E, W\}$.

Definition 1. Multiagent system (2) is said to achieve bounded consensus if for any initial state values $x_{i}\left(t_{0}\right)$ and $v_{i}\left(t_{0}\right)$, it follows that

$$
\left\{\begin{array}{l}
\sup \lim _{t \longrightarrow \infty}\left|x_{i}(t)-x_{j}(t)\right| \leq \varepsilon, \\
\sup \lim _{t \longrightarrow \infty}\left|v_{i}(t)-v_{j}(t)\right| \leq \xi,
\end{array}\right.
$$

where $\varepsilon$ and $\xi$ are constants.

Definition 2. Multiagent system (2) is said to achieve dynamic following consensus if for any initial state values $x_{i}\left(t_{0}\right)$ and $v_{i}\left(t_{0}\right)$, it follows that

$$
\left\{\begin{array}{l}
\lim _{t \rightarrow \infty} x_{i}(t)=x_{r}(t), \\
\lim _{t \longrightarrow \infty} v_{i}(t)=v_{r}(t),
\end{array}\right.
$$

where $x_{r}(t)$ and $v_{r}(t)$ are the position and velocity of the leader.

Lemma 1 (see [30]). Consider a polynomial $\mathrm{Q}(z)=z^{2}+(m+\mathbf{i} n) z+p+\mathbf{i} q$, where $m, n, p$, and $q$ are real constants; then, $Q(z)$ is Hurwitz stable if and only if $m>0$ and $m n q+m^{2} p-q^{2}>0$.

Lemma 2 (see [31]). All the roots of the equation $s^{2}+a s+b=0$, where $a, b \in C$, are within the unit circle if 
and only if all the roots of the equation $(a+b+1) z^{2}+2(1-$ b) $z+b-a+1=0$ are in the open left half plane.

Lemma 3 (see [13]). Laplacian matrix $L \in R^{n \times n}$ has a simple eigenvalue 0 , and all the nonzero eigenvalues have positive real parts if and only if the digraph has a spanning tree. In addition, $L \mathbf{1}_{n}=\mathbf{0}_{n}$, and there exists a nonnegative vector $q \in R^{n \times 1}$ satisfying $q^{T} L=\mathbf{0}_{n}^{T}$ and $q^{T} \mathbf{1}_{n}=\mathbf{1}$.

Lemma 4 (see [32]). Let $A_{11}, A_{12}, A_{21}, A_{22} \in R^{n \times n}$ and $A=\left[\begin{array}{ll}A_{11} & A_{12} \\ A_{21} & A_{22}\end{array}\right]$. Then, $\operatorname{det}(A)=\operatorname{det}\left(A_{11} A_{22}-A_{12} A_{21}\right)$ if $A_{11}, A_{12}, A_{21}$, and $A_{22}$ commute pairwise.

\section{Problem Illuminations}

In the section, a dynamic leader-following consensus protocol via impulsive control with different intelligence degrees is designed and necessary and sufficient conditions for its convergence are also given.

3.1. Leader-Following Consensus Protocol via Impulsive Control with Different Intelligence Degrees. A leader-following and bounded consensus protocol is designed as

$$
u_{i}(t)=-\sum_{l=1}^{\infty}\left[p_{1} \sum_{j=1}^{n} \frac{\omega_{i}(t)}{\omega_{j}(t)} l_{i j} x_{j}(t)+p_{2} \sum_{j=1}^{n} \frac{\omega_{i}(t)}{\omega_{j}(t)} l_{i j} v_{j}(t)+p_{1} b_{i}(t)\left(x_{i}(t)-x_{r}(t)\right)+p_{2} b_{i}(t)\left(v_{i}(t)-v_{r}(t)\right)\right] \delta\left(t-t_{l}\right)
$$

where $\delta(\cdot)$ is Dirac impulsive function and the agents intelligence degrees is a set of $\left\{\omega_{1}(t), \ldots, \omega_{n}(t)\right\}$. The sampling interval is denoted as $h$ with $t_{l}-t_{l-1} \equiv h>0 . p_{1}$ and $p_{2}$ are feedback gains of position error and speed error, respectively. The $b(t)$ is a time-varying parameter, which is denoted as $b(t)=\left\{b_{1}(t), b_{2}(t), \ldots, b_{n}(t)\right\}$ with adjacency element $b_{i}(t)>0$ if agent $i$ is a neighbor of the leader; otherwise, $b_{i}(t)=0$. Especially, $x_{r}(t)$ and $v_{r}(t)$ are the position and speed of the virtual leader at $t$ time instance, respectively.
Remark 1. The system runs without any control input if $t \neq t_{l}$, and the control input is available at $t=t_{l}$ to update the information from its neighbors.

Remark 2. If there are multiple leaders in the multiagent system, which means position and speed $x_{r}(t)$ and $v_{r}(t)$ are replaced by $x_{r}^{q}(t)$ and $v_{r}^{q}(t), q>1$, then the system will achieve multiobject tracking under the leader-following protocol.

System (2) and consensus protocol (5) can be rewritten as follows:

$$
\left\{\begin{array}{l}
\dot{x}_{i}(t)=v_{i}(t), \\
\dot{v}_{i}(t)=0 \\
\Delta v_{i}\left(t_{l}\right)=-p_{1} \sum_{j=1}^{n} \frac{\omega_{i}\left(t_{l}\right)}{\omega_{j}\left(t_{l}\right)} l_{i j} x_{j}(t)-p_{2} \sum_{j=1}^{n} \frac{\omega_{i}\left(t_{l}\right)}{\omega_{j}\left(t_{l}\right)} l_{i j} v_{j}\left(t_{l}\right)-p_{1} b_{i}(t)\left(x_{i}\left(t_{i}\right)-x_{r}(t)\right)-p_{2} b_{i}(t)\left(v_{i}(t)-v_{r}(t)\right),
\end{array}\right.
$$

where $\Delta v_{i}\left(t_{l}\right)=v_{i}\left(t_{l}^{+}\right)-v_{i}\left(t_{l}\right)$ and $v_{i}(t)$ is left-continuous at $t=t_{l}$. Let $\widehat{x}_{i}(t)=x_{i}(t)-x_{r}(t)$ and $\widehat{v}_{i}(t)=v_{i}(t)-v_{r}(t)$.
Since $\sum_{i=1}^{n} l_{i j} x_{r}(t)=0, L x_{r}(t)=\mathbf{0}_{n}$, and $\sum_{i=1}^{n} l_{i j} v_{r}(t)=0$, $L v_{r}(t)=\mathbf{0}_{n}$, the system (6) could be written as

$$
\left\{\begin{array}{l}
\dot{\hat{x}}_{i}(t)=\widehat{v}_{i}(t), \\
\dot{\widehat{v}}_{i}(t)=0, \\
\Delta \widehat{v}_{i}\left(t_{l}\right)=-p_{1} \sum_{j=1}^{n} \frac{\omega_{i}\left(t_{l}\right)}{\omega_{j}\left(t_{l}\right)} l_{i j} \widehat{x}_{j}\left(t_{l}\right)-p_{2} \sum_{j=1}^{n} \frac{\omega_{i}\left(t_{l}\right)}{\omega_{j}\left(t_{l}\right)} l_{i j} \widehat{v}_{j}\left(t_{l}\right)-p_{1} b_{i}\left(t_{l}\right) \widehat{x}_{i}\left(t_{l}\right)-p_{2} b_{i}\left(t_{l}\right) \widehat{v}_{i}(t) .
\end{array}\right.
$$


From (7), one has

$$
\left\{\begin{array}{l}
\widehat{x}_{i}\left(t_{l+1}\right)=\widehat{x}_{i}\left(t_{l}\right)+h \widehat{v}_{i}\left(t_{l}^{+}\right) \\
\widehat{v}_{i}\left(t_{l+1}\right)=\widehat{v}_{i}\left(t_{l}^{+}\right) \\
\widehat{v}_{i}\left(t_{l}^{+}\right)=\widehat{v}_{i}\left(t_{l}\right)-p_{1} \sum_{j=1}^{n} \frac{\omega_{i}\left(t_{l}\right)}{\omega_{j}\left(t_{l}\right)} l_{i j} \widehat{x}_{j}\left(t_{l}\right)-p_{2} \sum_{j=1}^{n} \frac{\omega_{i}\left(t_{l}\right)}{\omega_{j}\left(t_{l}\right)} l_{i j} \widehat{v}_{j}\left(t_{l}\right)-p_{1} b_{i}\left(t_{l}\right) \widehat{x}_{i}\left(t_{l}\right)-p_{2} b_{i}\left(t_{l}\right) \widehat{v}_{i}\left(t_{l}\right)
\end{array}\right.
$$

where $h$ is the sampling interval.

Therefore, states $\widehat{x}_{i}(t)$ and $\widehat{x}_{j}(t)$ under protocol (5) can be described, respectively, as follows:

$$
\left\{\begin{array}{l}
\widehat{x}_{i}\left(t_{l+1}\right)=\widehat{x}_{i}\left(t_{l}\right)+\left[\widehat{v}_{i}\left(t_{l}\right)-p_{1} \sum_{j=1}^{n} \frac{\omega_{i}\left(t_{l}\right)}{\omega_{j}\left(t_{l}\right)} l_{i j} \widehat{x}_{j}\left(t_{l}\right)-p_{2} \sum_{j=1}^{n} \frac{\omega_{i}\left(t_{l}\right)}{\omega_{j}\left(t_{l}\right)} l_{i j} \widehat{v}_{j}\left(t_{l}\right)-p_{1} b_{i}\left(t_{l}\right) \widehat{x}_{i}\left(t_{l}\right)-p_{2} b_{i}\left(t_{l}\right) \widehat{v}_{i}\left(t_{l}\right)\right] h, \\
\widehat{v}_{i}\left(t_{l+1}\right)=\widehat{v}_{i}\left(t_{l}\right)-p_{1} \sum_{j=1}^{n} \frac{\omega_{i}\left(t_{l}\right)}{\omega_{j}\left(t_{l}\right)} l_{i j} \widehat{x}_{j}\left(t_{l}\right)-p_{2} \sum_{j=1}^{n} \frac{\omega_{i}\left(t_{l}\right)}{\omega_{j}\left(t_{l}\right)} l_{i j} \widehat{v}_{j}\left(t_{l}\right)-p_{1} b_{i}\left(t_{l}\right) \widehat{x}_{i}\left(t_{l}\right)-p_{2} b_{i}(t) \widehat{v}_{i}\left(t_{l}\right) .
\end{array}\right.
$$

Let $\widehat{x}\left(t_{l}\right)=\left[\widehat{x}_{1}\left(t_{l}\right), \ldots, \widehat{x}_{n}\left(t_{l}\right)\right]^{T}, \widehat{v}\left(t_{l}\right)=\left[\widehat{v}_{1}\left(t_{l}\right), \ldots, \widehat{v}_{n}\left(t_{l}\right)\right]$ and

$$
B\left(t_{l}\right)=\left[\begin{array}{llll}
b_{1}\left(t_{l}\right) & & & \\
& b_{2}\left(t_{l}\right) & & \\
& & \ddots & \\
& & & b_{n}\left(t_{l}\right)
\end{array}\right] \text {, }
$$

then equation (9) can be equivalently expressed as follows:

$$
\left(\begin{array}{c}
\hat{x}\left(t_{l+1}\right) \\
\widehat{v}\left(t_{l+1}\right)
\end{array}\right)=P\left(t_{l}\right)\left(\begin{array}{c}
\hat{x}\left(t_{l}\right) \\
\widehat{v}\left(t_{l}\right)
\end{array}\right),
$$

where

$$
\begin{aligned}
P\left(t_{l}\right)= & \left(I_{n}-h p_{1}\left(\widehat{L}\left(t_{l}\right)+B\left(t_{l}\right)\right) h I_{n}-h p_{2}\left(\widehat{L}\left(t_{l}\right)+B\left(t_{l}\right)\right)\right. \\
& \left.-p_{1}\left(\widehat{L}\left(t_{l}\right)+B\left(t_{l}\right)\right) I_{n}-p_{2}\left(\widehat{L}\left(t_{l}\right)+B\left(t_{l}\right)\right)\right) .
\end{aligned}
$$

Let

$$
\begin{gathered}
X\left(t_{l}\right)=\left[\begin{array}{lll}
\omega_{1}\left(t_{l}\right) & & \\
& \ddots & \\
& & \omega_{n}\left(t_{l}\right)
\end{array}\right], \\
X\left(t_{l}\right)^{-1}=\left[\begin{array}{lll}
\frac{1}{\omega_{1}\left(t_{l}\right)} & & \\
& \ddots & \\
& & \frac{1}{\omega_{n}\left(t_{l}\right)}
\end{array}\right] .
\end{gathered}
$$

The linear transformation of matrix $\widehat{L}\left(t_{l}\right)=X\left(t_{l}\right) L X\left(t_{l}\right)^{-1}$ is as follows:

$$
\widehat{L}\left(t_{l}\right)=\left[\begin{array}{cccc}
l_{11} & \frac{\omega_{1}\left(t_{l}\right)}{\omega_{2}\left(t_{l}\right)} l_{12} & \cdots & \frac{\omega_{1}\left(t_{l}\right)}{\omega_{n}\left(t_{l}\right)} l_{1 n} \\
\frac{\omega_{2}\left(t_{l}\right)}{\omega_{1}\left(t_{l}\right)} l_{21} & l_{22} & \cdots & \frac{\omega_{2}\left(t_{l}\right)}{\omega_{n}\left(t_{l}\right)} l_{2 n} \\
\vdots & \vdots & \ddots & \vdots \\
\frac{\omega_{n}\left(t_{l}\right)}{\omega_{1}\left(t_{l}\right)} l_{n 1} & \frac{\omega_{n}\left(t_{l}\right)}{\omega_{2}\left(t_{l}\right)} l_{n 2} & \cdots & l_{n n}
\end{array}\right] .
$$

Furthermore,

$$
\widehat{L}\left(t_{l}\right)+B\left(t_{l}\right)=\left[\begin{array}{cccc}
l_{11}+b_{1}\left(t_{l}\right) & \frac{\omega_{1}\left(t_{l}\right)}{\omega_{2}\left(t_{l}\right)} l_{12} & \ldots & \frac{\omega_{1}\left(t_{l}\right)}{\omega_{n}\left(t_{l}\right)} l_{1 n} \\
\frac{\omega_{2}\left(t_{l}\right)}{\omega_{1}\left(t_{l}\right)} l_{21} & l_{22}+b_{2}\left(t_{l}\right) & \cdots & \frac{\omega_{2}\left(t_{l}\right)}{\omega_{n}\left(t_{l}\right)} l_{2 n} \\
\vdots & \vdots & \ddots & \vdots \\
\frac{\omega_{n}\left(t_{l}\right)}{\omega_{1}\left(t_{l}\right)} l_{n 1} & \frac{\omega_{n}\left(t_{l}\right)}{\omega_{2}\left(t_{l}\right)} l_{n 2} & \cdots & l_{n n}+b_{n}\left(t_{l}\right)
\end{array}\right] .
$$




\subsection{Main Results}

Theorem 1. Matrix $P\left(t_{l}\right)$ has two eigenvalues 1 , and the other eigenvalues lie in the unit circle, if and only if the digraph $G$ contains a spanning tree and

$$
\begin{aligned}
& 0<p_{2}<\frac{2 \operatorname{Re}\left(\lambda_{i}\left(t_{l}\right)\right)}{\left|\lambda_{i}\left(t_{l}\right)\right|^{2}}, \\
& 0<h<\min _{\lambda_{i}\left(t_{l}\right) \neq 0} \frac{-2 p_{2}^{3}\left|\lambda_{i}\left(t_{l}\right)\right|^{4}+4 p_{2}^{2} \operatorname{Re}\left(\lambda_{i}\left(t_{l}\right)\right)\left|\lambda_{i}\left(t_{l}\right)\right|^{2}}{\left[p_{2}^{2}\left|\lambda_{i}\left(t_{l}\right)\right|^{4}+4\left(\operatorname{Im}\left(\lambda_{i}\left(t_{l}\right)\right)\right)^{2}\right] p_{1}},
\end{aligned}
$$

where the eigenvalues of matrix $\widehat{L}\left(t_{l}\right)+B\left(t_{l}\right)$ are $\lambda_{i}\left(t_{l}\right)$ and the real and imaginary parts of the matrix $\widehat{L}\left(t_{l}\right)+B\left(t_{l}\right)$ are $\operatorname{Re}\left(\lambda_{i}\left(t_{l}\right)\right)$ and $\operatorname{Im}\left(\lambda_{i}\left(t_{l}\right)\right)$.

Proof 1. The characteristic polynomial of $P\left(t_{l}\right)$ is given by

$$
\begin{aligned}
\operatorname{det}\left(s I_{2 n}-P\left(t_{l}\right)\right) & =\operatorname{det}\left[\begin{array}{cc}
s I_{n}-I_{n}+h p_{1}\left(\widehat{L}\left(t_{l}\right)+B\left(t_{l}\right)\right) & -h I_{n}+h p_{2}\left(\widehat{L}\left(t_{l}\right)+B\left(t_{l}\right)\right) \\
p_{1}\left(\widehat{L}\left(t_{l}\right)+B\left(t_{l}\right)\right) & s I_{n}-I_{n}+p_{2}\left(\widehat{L}\left(t_{l}\right)+B\left(t_{l}\right)\right)
\end{array}\right] \\
& =\operatorname{det}\left[s^{2} I_{n}+s\left(h p_{1}\left(\widehat{L}\left(t_{l}\right)+B\left(t_{l}\right)\right)+p_{2}\left(\widehat{L}\left(t_{l}\right)+B\left(t_{l}\right)\right)-2 I_{n}\right)+I_{n}-p_{2}\left(\widehat{L}\left(t_{l}\right)+B\left(t_{l}\right)\right)\right] \\
& =\prod_{i=1}^{n}\left[s^{2}+s\left(h p_{1} \lambda_{i}\left(t_{l}\right)+p_{2} \lambda_{i}\left(t_{l}\right)-2\right)+1-p_{2} \lambda_{i}\left(t_{l}\right)\right] .
\end{aligned}
$$

Let

$$
s^{2}+s\left(h p_{1} \lambda_{i}\left(t_{l}\right)+p_{2} \lambda_{i}\left(t_{l}\right)-2\right)+1-p_{2} \lambda_{i}\left(t_{l}\right) .
$$

By Lemma 2, the eigenvalues of matrix $P\left(t_{l}\right)$ lie in the unit circle if and only if all the roots of

$$
z^{2}+\frac{2 p_{2}}{h p_{1}} z+\frac{4\left(1 / \lambda_{i}\left(t_{l}\right)\right)-2 p_{2}-h p_{1}}{h p_{1}}=0,
$$

are in the open left half plane.

Define $Q_{i}(z)$ as

$$
\begin{aligned}
Q_{i}(z)= & z^{2}+\frac{2 p_{2}}{h p_{1}} z+\frac{4\left(1 / \lambda_{i}\left(t_{l}\right)\right)-2 p_{2}-h p_{1}}{h p_{1}} \\
= & z^{2}+\frac{2 p_{2}}{h p_{1}} z+\frac{\left(-2 p_{2}-h p_{1}\right)\left|\lambda_{i}\left(t_{l}\right)\right|^{2}+4 \operatorname{Re}\left(\lambda_{i}\left(t_{l}\right)\right)}{h p_{1}\left|\lambda_{i}\left(t_{l}\right)\right|^{2}} \\
& -\frac{4 \operatorname{Im}\left(\lambda_{i}\left(t_{l}\right)\right)}{h p_{1}\left|\lambda_{i}\left(t_{l}\right)\right|^{2}} \mathbf{i} .
\end{aligned}
$$

Combined with Lemma 1 that $Q_{i}(z)$ is Hurwitz stable, if and only if $2 p_{2} / h p_{1}>0$, and $\left(4 p_{2}^{2}\left|\lambda_{i}\left(t_{l}\right)\right|^{2}\left[\left(-2 p_{2}-h p_{1}\right)\right.\right.$ $\left.\left.\left|\lambda_{i}\left(t_{l}\right)\right|^{2}+4 \operatorname{Re}\left(\lambda_{i}\left(t_{l}\right)\right)\right]-16 h p_{1}\left(\operatorname{Im}\left(\lambda_{i}\left(t_{l}\right)\right)\right)^{2}\right) /\left(h^{3} p_{1}^{3}\right.$ $\left.\left|\lambda_{i}\left(t_{l}\right)\right|^{4}\right)>0$, it is indicated that

$$
\begin{aligned}
& 4 p_{2}^{2}\left|\lambda_{i}\left(t_{l}\right)\right|^{2}\left[\left(-2 p_{2}-h p_{1}\right)\left|\lambda_{i}\left(t_{l}\right)\right|^{2}+4 \operatorname{Re}\left(\lambda_{i}\left(t_{l}\right)\right)\right] \\
& -16 h p_{1}\left(\operatorname{Im}\left(\lambda_{i}\left(t_{l}\right)\right)\right)^{2}>0 .
\end{aligned}
$$

Then, one has

$$
0<h<\min _{\lambda_{i}\left(t_{l}\right) \neq 0} \frac{-2 p_{2}^{3}\left|\lambda_{i}\left(t_{l}\right)\right|^{4}+4 p_{2}^{2} \operatorname{Re}\left(\lambda_{i}\left(t_{l}\right)\right)\left|\lambda_{i}\left(t_{l}\right)\right|^{2}}{\left[p_{2}^{2}\left|\lambda_{i}\left(t_{l}\right)\right|^{4}+4\left(\operatorname{Im}\left(\lambda_{i}\left(t_{l}\right)\right)\right)^{2}\right] p_{1}}
$$

Note that

$$
\begin{aligned}
& -2 p_{2}^{3}\left|\lambda_{i}\left(t_{l}\right)\right|^{4}+4 p_{2}^{2} \operatorname{Re}\left(\lambda_{i}\left(t_{l}\right)\right)\left|\lambda_{i}\left(t_{l}\right)\right|^{2}>0, \\
& 0<p_{2}<\frac{2 \operatorname{Re}\left(\lambda_{i}\left(t_{l}\right)\right)}{\left|\lambda_{i}\left(t_{l}\right)\right|^{2}} .
\end{aligned}
$$

By Lemma 3, $P\left(t_{l}\right)$ has two eigenvalues 1 , and the other eigenvalues lie in the unit circle if and only if the digraph has a spanning tree, and

$$
\begin{aligned}
& 0<p_{2}<\frac{2 \operatorname{Re}\left(\lambda_{i}\left(t_{l}\right)\right)}{\left|\lambda_{i}\left(t_{l}\right)\right|^{2}}, \\
& 0<h<\min _{\lambda_{i}\left(t_{l}\right) \neq 0} \frac{-2 p_{2}^{3}\left|\lambda_{i}\left(t_{l}\right)\right|^{4}+4 p_{2}^{2} \operatorname{Re}\left(\lambda_{i}\left(t_{l}\right)\right)\left|\lambda_{i}\left(t_{l}\right)\right|^{2}}{\left[p_{2}^{2}\left|\lambda_{i}\left(t_{l}\right)\right|^{4}+4\left(\operatorname{Im}\left(\lambda_{i}\left(t_{l}\right)\right)\right)^{2}\right] p_{1}} .
\end{aligned}
$$

This completes the proof.

If there is not a leader-following mechanism in system (2), the multiagent system becomes a general consensus system. That is, letting

$$
\begin{aligned}
& B_{0}\left(t_{l}\right)=\left[\begin{array}{llll}
0 & & & \\
& 0 & & \\
& & \ddots & \\
& & & 0
\end{array}\right], \\
& \widehat{L}\left(t_{l}\right)+B_{0}\left(t_{l}\right)=\widehat{L}\left(t_{l}\right),
\end{aligned}
$$




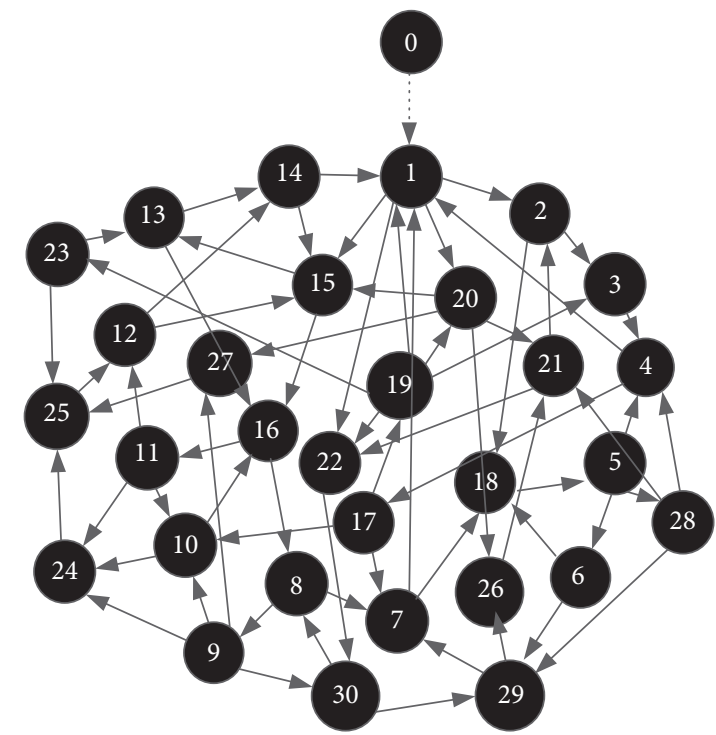

Figure 1: The directed topology with 30 agents, where agent 0 is the virtual leader.

then

$$
P_{0}\left(t_{l}\right)=\left(\begin{array}{cc}
I_{N}-h p_{1} \widehat{L}\left(t_{l}\right) & h I_{N}-h p_{2} \widehat{L}\left(t_{l}\right) \\
-p_{1} \widehat{L}\left(t_{l}\right) & I_{N}-p_{2} \widehat{L}\left(t_{l}\right)
\end{array}\right),
$$

where $P_{0}(t)$ is the state space matrix such that there is no leader-following system (2).

Remark 3. $\widehat{L}\left(t_{l}\right)$ has one right and left eigenvector $\widehat{p}\left(t_{l}\right)=$ $\left[\omega_{1}\left(t_{l}\right), \ldots, \omega_{n}\left(t_{l}\right)\right]^{T}$ and $\widehat{q}\left(t_{l}\right) \in R^{1 \times n}$ associated with the eigenvalue 0 , respectively, such that $\widehat{L}\left(t_{l}\right) \widehat{p}\left(t_{l}\right)=\mathbf{0}$ and $\widehat{q}\left(t_{l}\right) \widehat{L}\left(t_{l}\right)=\mathbf{0}^{T}$ with $\widehat{q}\left(t_{l}\right) \widehat{p}\left(t_{l}\right)=1$ based on Lemma 3 .

If there is no virtual leader, this system can only achieve bounded consensus. Then, we can achieve obstacle avoidance by changing the intelligent degree of agents. For illuminating the multiconsensus convergence of multiagent systems, Theorem 2 is given to prove the final convergent states.

Theorem 2. Under impulsive control protocol (5), as $t_{l} \longrightarrow \infty$ and there is no leader-following, multiagent system (2) of the states of agents is

$$
\begin{aligned}
& x_{i}\left(t_{l}\right) \longrightarrow \omega_{i}\left(t_{l}\right) \hat{q}\left(t_{l}\right)\left[x\left(t_{0}\right)+v\left(t_{0}\right) t_{l}\right], \\
& v_{i}\left(t_{l}\right) \longrightarrow \omega_{i}\left(t_{l}\right) \hat{q}\left(t_{l}\right) v\left(t_{0}\right),
\end{aligned}
$$

if and only if the digraph contains a spanning tree and equations (16) and (17) hold.

Proof 2. Sufficiency: the matrix $P_{0}\left(t_{l}\right)$ has one right and generalized right eigenvector $\varphi_{1}\left(t_{l}\right)=[1,0]^{T} \otimes \widehat{p}\left(t_{l}\right)$ and $\varphi_{2}\left(t_{l}\right)=[0,1 / h]^{T} \otimes \widehat{p}\left(t_{l}\right)$ associated with the eigenvalue 1 , respectively. Moreover, $P_{0}\left(t_{l}\right)$ has one left and generalized left eigenvector $\gamma_{1}\left(t_{l}\right)=[1,0] \otimes \widehat{q}\left(t_{l}\right)$ and $\gamma_{2}\left(t_{l}\right)=[0$, $h] \otimes \widehat{q}\left(t_{l}\right)$ associated with the eigenvalue 1 , respectively. There exist nonsingular matrices $\Phi\left(t_{l}\right)=\left[\varphi_{1}\left(t_{l}\right), \ldots\right.$, $\left.\varphi_{2 n}\left(t_{l}\right)\right]$ and $\Gamma\left(t_{l}\right)=\left[\gamma_{1}^{T}\left(t_{l}\right), \ldots, \gamma_{2 n}^{T}\left(t_{l}\right)\right]^{T}$ such that $P_{0}\left(t_{l}\right)$ can be written in a Jordan canonical form as

$$
P_{0}\left(t_{l}\right)=\Phi\left(t_{l}\right)\left[\begin{array}{ll}
J_{0} & \\
& J
\end{array}\right] \Gamma\left(t_{l}\right),
$$

where $J_{0}=\left[\begin{array}{ll}1 & 1 \\ & 1\end{array}\right]$ and $\Phi\left(t_{l}\right) \Gamma\left(t_{l}\right)=I_{2 n}$.

Hence, one can obtain that

$$
P_{0}\left(t_{l}\right)^{l}=\Phi\left(t_{l}\right)\left[\begin{array}{ll}
J_{0}^{l} & \\
& \\
& J^{l}
\end{array}\right] \Gamma\left(t_{l}\right),
$$

where $J_{0}^{l}=\left[\begin{array}{ll}1 & l \\ & 1\end{array}\right]$. Denote that

$$
\prod(l) \triangleq\left[\varphi_{1}\left(t_{l}\right), \varphi_{2}\left(t_{l}\right)\right] J_{0}^{l}\left[\gamma_{1}^{T}\left(t_{l}\right), \gamma_{2}^{T}\left(t_{l}\right)\right]^{T}=\left[\begin{array}{cc}
\omega_{1}\left(t_{l}\right) & t_{l} \omega_{1}\left(t_{l}\right) \\
\vdots & \vdots \\
\omega_{n}\left(t_{l}\right) & t_{l} \omega_{n}\left(t_{l}\right) \\
0 & \omega_{1}\left(t_{l}\right) \\
\vdots & \vdots \\
0 & \omega_{n}\left(t_{l}\right)
\end{array}\right] \otimes \widehat{q}\left(t_{l}\right)
$$




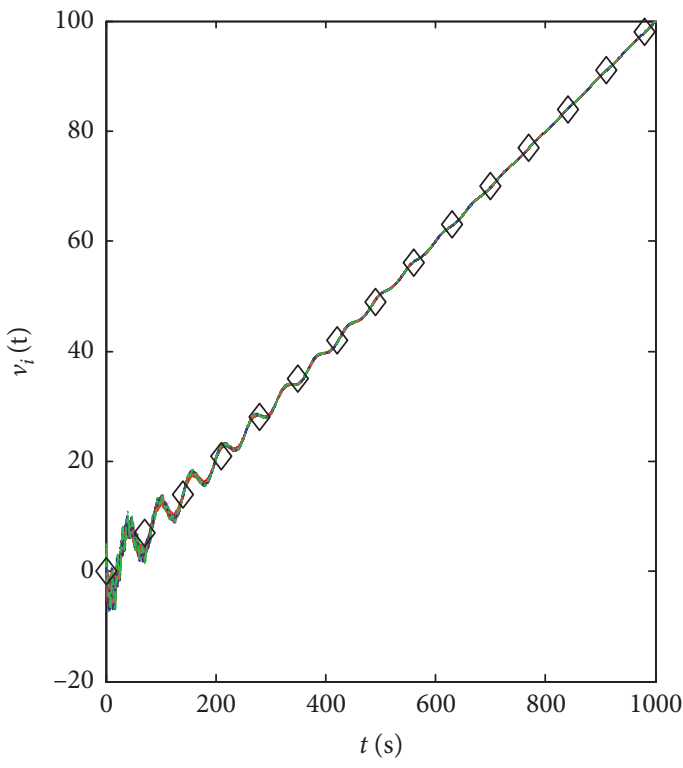

(a)

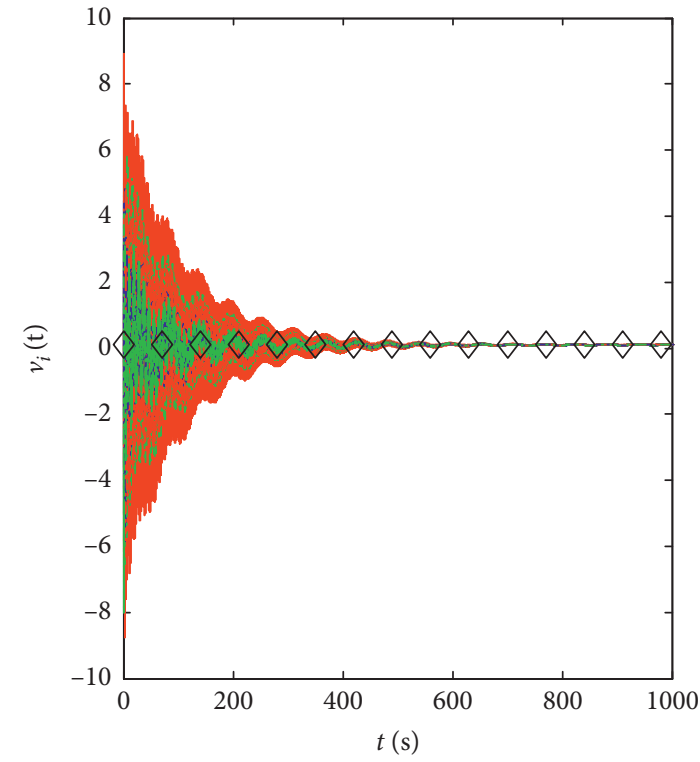

(b)

FIgURE 2: Trajectory of leader-following bounded consensus with $h=0.5$.

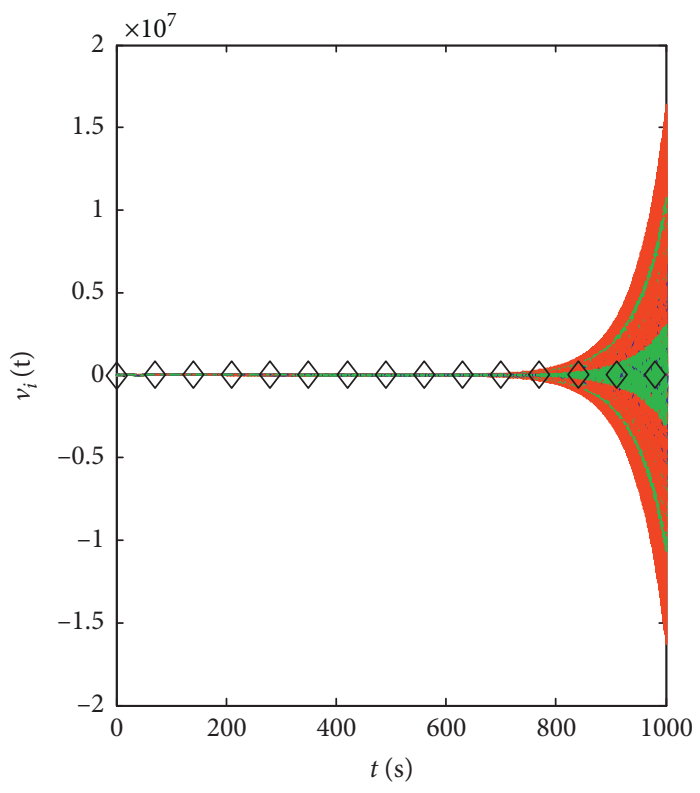

(a)

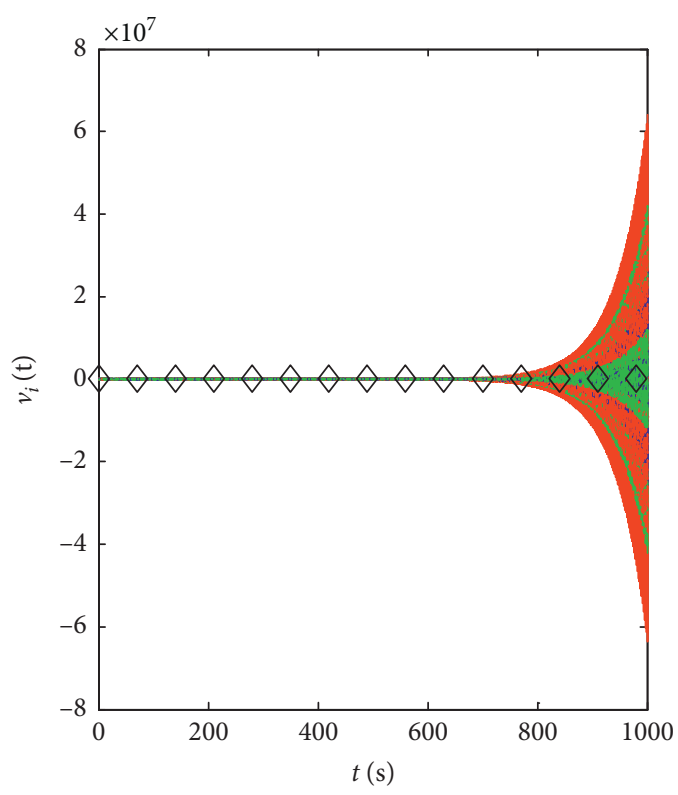

(b)

FIgURE 3: Trajectory of leader-following bounded consensus with $h=0.504$.

where $t_{l}=l h$.

Letting $\bar{\Phi}\left(t_{l}\right)=\left[\varphi_{3}\left(t_{l}\right), \ldots, \varphi_{2 n}\left(t_{l}\right)\right]$ $\bar{\Gamma}\left(t_{l}\right)=\left[\gamma_{3}\left(t_{l}\right), \ldots, \gamma_{2 n}\left(t_{l}\right)\right]$, then

$\lim _{l \longrightarrow \infty}\left\|P_{0}\left(t_{l}\right)^{l}-\prod(l)\right\|=\lim _{l \longrightarrow \infty}\left\|\bar{\Phi}\left(t_{l}\right) J^{l} \bar{\Gamma}\left(t_{l}\right)\right\|=0$.

As $t_{l} \longrightarrow \infty$, we have

$$
\begin{aligned}
& x_{i}\left(t_{l}\right) \longrightarrow \omega_{i}\left(t_{l}\right) \hat{q}\left(t_{l}\right)\left[x\left(t_{0}\right)+v\left(t_{0}\right) t_{l}\right], \\
& v_{i}\left(t_{l}\right) \longrightarrow \omega_{i}\left(t_{l}\right) \hat{q}\left(t_{l}\right) v\left(t_{0}\right) .
\end{aligned}
$$
and
Necessity: if the dynamic bounded consensus is solved in protocol (5), it holds that $\lim _{l \longrightarrow \infty} P_{0}\left(t_{l}\right)^{l}$ has rank two; that is, $P_{0}\left(t_{l}\right)^{l}$ has two eigenvalues 1 and the other eigenvalues lie in the unit circle. Based on Theorem 1, the digraph has a spanning tree, and (16) and (17) hold.

This completes the proof.

Remark 4. From equations (28) and (29), we know that if we change the dynamic behaviour of agents by regulating the intelligent degree of agents, the dynamic characteristic of the 


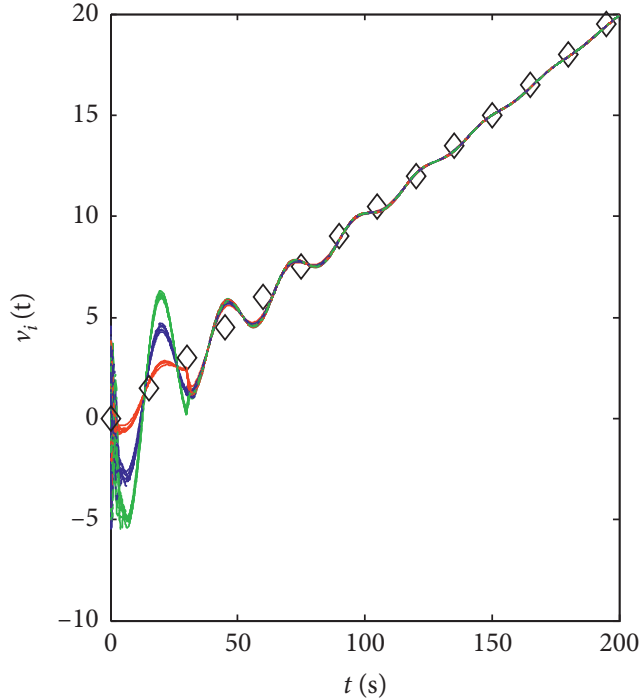

(a)

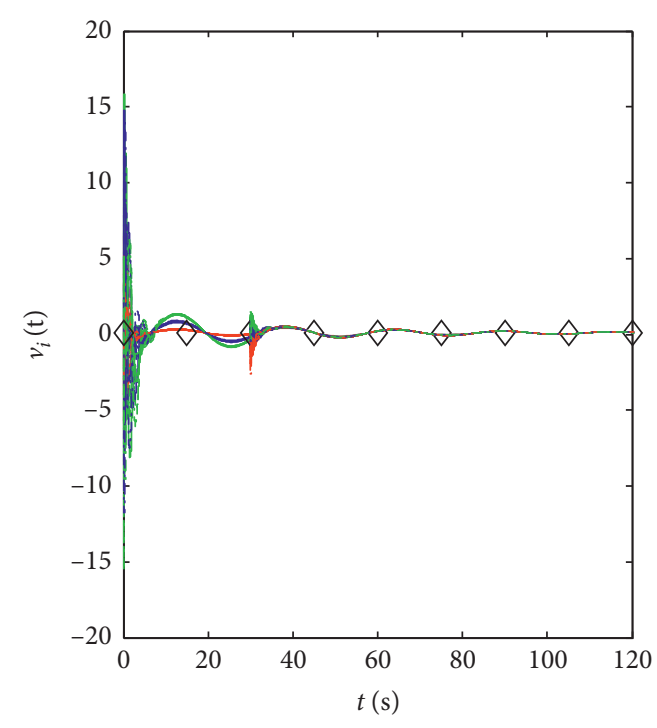

(b)

FIgURE 4: Trajectory of multiconsensus to consensus dynamic evolution by changed intelligence degrees of some agents with $h=0.1$, $p_{1}=0.4$, and $p_{2}=0.3$.

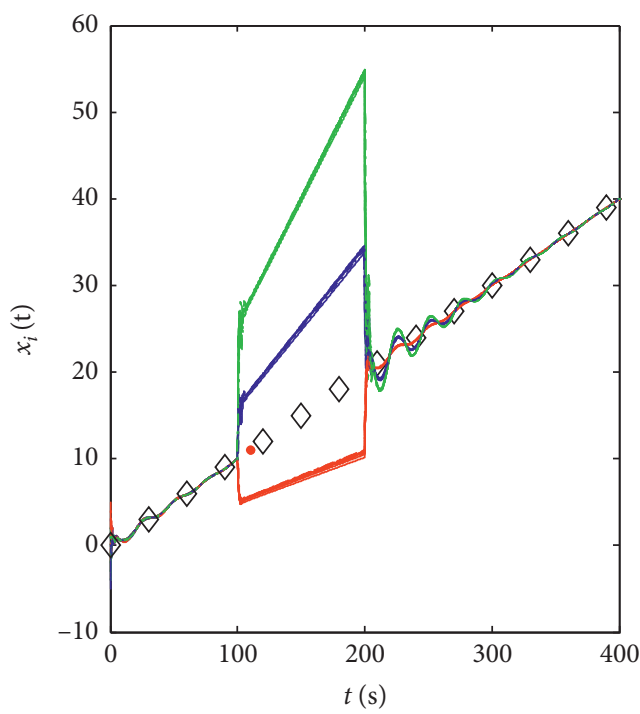

(a)

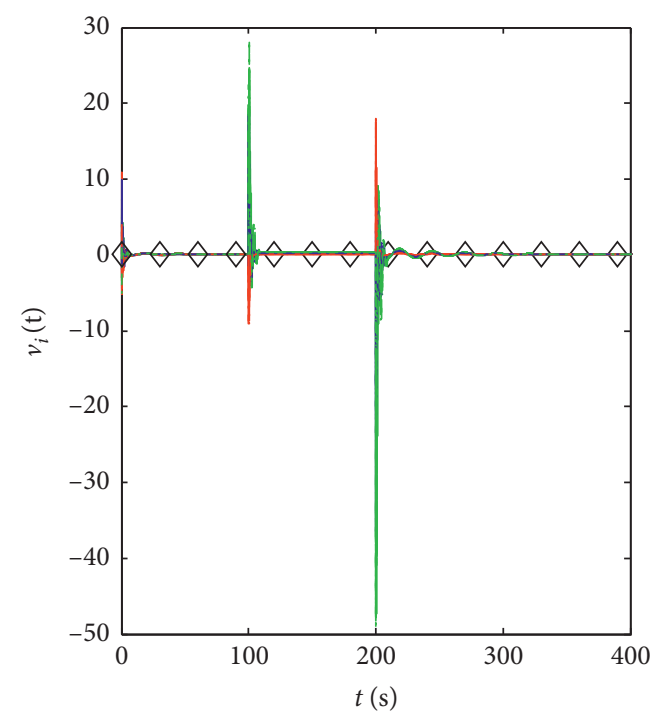

(b)

Figure 5: Trajectory of consensus to multiconsensus dynamic evolution and obstacle avoidance by changed intelligences degrees with $h=0.1, p_{1}=0.4$, and $p_{2}=0.3$, and the red point is the obstacle in the trajectory of the virtual leader.

agents at each moment can be obtained by equations (28) and (29).

\section{Numerical Example}

In this section, an illustrative example is given to demonstrate the correctness of the theoretical results.

Example 1. Consider the multiagent system with 30 agents as shown in Figure 1.
The dynamic equation of the virtual leader are $x_{0}(t)=$ $0.1 * t$ and $v_{0}(t)=0.001$ in simulation. Choose $p_{1}=0.4$ and $p_{2}=0.3$, and the result is $0<h<0.5035$ via Theorem 1 . Therefore, choose $h=0.5$ and $h=0.504$, and the intelligent degrees belong to $\omega(t) \in(5,5.5)$; the results are shown in Figures 2 and 3 .

Choose the intelligence degrees from $\omega(t) \in(3,3.5)$, $\omega(t) \in(10,10.5)$, and $\omega(t) \in(16,16.5)$ as initial values, respectively. Meanwhile, there is a virtual leader in the system. When time is 30 seconds, the intelligence degrees of agents become $\omega(t) \in(3,3.5)$. The multiconsensus to 
consensus dynamic evolution is realized, as shown in Figure 4.

For showing obstacle avoidance through the dynamic evolution from consensus to multiconsensus when initial intelligence degrees are switched from $\omega(t) \in(3,3.5)$ to choose the intelligence degrees from $\omega(t) \in(3,3.5)$, it is shown that $\omega(t) \in(10,10.5)$ and $\omega(t) \in(16,16.5)$. Meanwhile, there is no virtual leader within $t \in(100,200)$, and details are shown in Figure 5.

In Figure 5, if the anticipate trajectory of the virtual leader is hindered by the red and round obstacle, agent 1 gives up to connect the virtual leader. Then, all agents no longer follow the virtual leader and change their intelligence degrees. As a result, the multiagent system will run according to multiconsensus behaviour. The virtual leader will not reestablish communication with agent 1 until all agents have avoided the obstacle. Then, the system achieves leader-following and bounded consensus again.

\section{Conclusions}

In this paper, the leader-following consensus and multiconsensus problem has been considered for continuous-time second-order multiagent systems. The system could achieve consensus and multiconsensus commutative evolution by regulating the intelligence degrees. An impulsive control protocol with different intelligence degrees is proposed to perform obstacle avoidance, and some necessary and sufficient conditions for leader-following bounded consensus and multiconsensus have been obtained. It is found that the consensus and multiconsensus leader-following are critically dependent on the sampling interval, control gains, and the intelligence degrees. In the future, one of our works will consider multiagent systems achieving obstacle avoidance automatically.

\section{Data Availability}

The data used to support the findings of the study are available from the corresponding author upon request.

\section{Conflicts of Interest}

The authors declare that there are no conflicts of interest regarding the publication of this paper.

\section{Acknowledgments}

This work was supported by the National Nature Science Foundation of China under Grants 62063011 and 61922037 and the Scientific Research Fund of Yunnan Education Department under Grants 2020J0067 and 2019J0046.

\section{References}

[1] H. M. Lee, S. D. Min, M. H. Choi et al., "Multi-agent system for fault tolerance in wireless sensor networks," KSII Transactions on Internet \& Information Systems, vol. 10, no. 3, pp. 1321-1332, 2016.
[2] L. Zou, Q. Ding, and R. Zhou, "Distributed cooperative guidance for multiple heterogeneous networked missiles," Journal of Beijing University of Aeronautics and Astronautics, vol. 36, no. 12, pp. 1432-1435, 2010.

[3] H. Xiao and C. L. P. Chen, "Leader-follower consensus multi-robot formation control using neurodynamic-optimization-based nonlinear model predictive control," IEEE Access, vol. 7, pp. 43581-43590, 2019.

[4] S. B. Wang, J. Na, and Y. S. Xing, "Adaptive optimal parameter estimation and control of servo mechanisms: theory and experiment," IEEE Transactions on Industrial Electronics, 2019.

[5] S. B. Wang and J. Na, "Parameter estimation and adaptive control for servo mechanisms with friction compensation," IEEE Transactions on Industrial Informatics, vol. 16, no. 11, pp. 6816-6825, 2020.

[6] S. B. Wang, L. Tao, Q. Chen, J. Na, and X. M. Ren, "USDEBased sliding model control for servo mechanisms with unknown system dynamics," IEEE/ASME Transactions on Mechatronics, vol. 25, no. 2, pp. 1056-1066, 2020.

[7] Q. Chen, X. Q. Yu, M. X. Sun, C. Wu, and Z. J. Fu, “Adaptive repetitive learning control of PMSM servo systems with bounded nonparametric uncertainties: theory and experiments," IEEE Transactions on Industrial Electronics, 2020.

[8] R. Olfati-Saber and R. M. Murray, "Consensus problems in networks of agents with switching topology and time-delays," IEEE Transactions on Automatic Control, vol. 49, no. 9, pp. 1520-1533, 2004.

[9] W. Ren and R. W. Beard, "Consensus seeking in multi-agent systems under dynamically changing interaction topologies," IEEE Transactions on Automatic Control, vol. 50, no. 5, pp. 655-661, 2005.

[10] F. Sun, Y. Shen, J. Kurths, and W. Zhu, "Mean-square consensus of multi-agent systems with noise and time delay via event-triggered control," Journal of the Franklin Institute, vol. 357, no. 9, pp. 5317-5339, 2020.

[11] Y. Cai, H. Zhang, Z. Gao, and S. Sun, “The distributed output consensus control of linear heterogeneous multi-agent systems based on event-triggered transmission mechanism under directed topology," Journal of the Franklin Institute, vol. 357, no. 6, pp. 3267-3298, 2020.

[12] Z.-W. Liu, Z.-H. Guan, X. Shen, and G. Feng, "Consensus of multi-agent networks with aperiodic sampled communication via impulsive algorithms using position-only measurements," IEEE Transactions on Automatic Control, vol. 57, no. 10, pp. 2639-2643, 2012.

[13] X. Pu, L. Zhao, and C. Xiong, "Weighted group consensus for discrete-time heterogeneous multi-agent systems in the cooperative-competitive network with time delays," IEEE Access, vol. 7, pp. 123679-123688, 2019.

[14] L. Ji, Q. Liu, and X. Liao, "On reaching group consensus for linearly coupled multi-agent networks," Information Sciences, vol. 287, pp. 1-12, 2014.

[15] Y. Shang, "Group consensus of multi-agent systems with switching topologies and stochastic inputs," Physics Letters A, vol. 377, no. 25-27, pp. 1582-1586, 2013.

[16] J. Almeida, C. Silvestre, and A. M. Pascoal, "Continuous-time consensus with discrete-time communications," Systems \& Control Letters, vol. 61, no. 7, pp. 788-796, 2012.

[17] Q. Chen, H. H. Shi, and M. X. Sun, "Echo state network based backstepping adaptive iterative learning control for strictfeedback systems: an error-tracking approach," IEEE Transactions on Cybernetics, vol. 50, no. 7, 2019.

[18] Y.-Y. Qian, L. Liu, and G. Feng, "Distributed event-triggered adaptive control for consensus of linear multi-agent systems 
with external disturbances," IEEE Transactions on Cybernetics, vol. 50, no. 5, pp. 2197-2208, 2020.

[19] G.-S. Han, D.-X. He, Z.-H. Guan, B. Hu, T. Li, and R.-Q. Liao, "Multi-consensus of multi-agent systems with various intelligences using switched impulsive protocols," Information Sciences, vol. 349-350, pp. 188-198, 2016.

[20] J. Sun and C. Liu, "Distributed fuzzy adaptive backstepping optimal control for nonlinear multi-missile guidance systems with input saturation," IEEE Transactions on Fuzzy Systems, vol. 3, p. 1, 2018

[21] B. Zhang, X. Sun, S. Liu, and X. Deng, “Adaptive differential evolution-based receding horizon control design for multiUAV Formation reconfiguration," International Journal of Control, Automation and Systems, vol. 17, no. 12, pp. 30093020, 2019.

[22] W. He, B. Zhang, Q. L. Han, F Qian, J Kurths, and J Cao, "Leader-following consensus of nonlinear multiagent systems with stochastic sampling," IEEE Transactions on Cybernetics, vol. 47, no. 2, pp. 327-338, 2017.

[23] W. Zhu, D. Wang, and Q. Zhou, "Leader-following consensus of multi-agent systems via adaptive event-based control," Journal of Systems Science and Complexity, vol. 32, no. 3, pp. 846-856, 2019.

[24] C.-E. Ren, C. L. P. Chen, T. Du, and Y. Guan, "Fuzzy adaptive leader-following consensus control for nonlinear multi-agent systems with unknown control directions," International Journal of Fuzzy Systems, vol. 21, no. 7, pp. 2066-2076, 2019.

[25] J. A. Douthwaite, S. Zhao, and L. S. Mihaylova, "Velocity obstacle approaches for multi-agent collision avoidance," Unmanned Systems, vol. 7, no. 1, pp. 55-64, 2019.

[26] Y. Guo, J. Zhou, and Y. Liu, "Distributed Lyapunov-based model predictive control for collision avoidance of multiagent formation," IET Control Theory \& Applications, vol. 12, no. 18, pp. 2569-2577, 2018.

[27] W. Zhang, Y. Ma, H. D. Zhao et al., "Obstacle avoidance path planning of intelligent mobile based on improved fireworksant colony hybrid algorithm," Control and Decision, vol. 34, no. 2, pp. 335-343, 2019.

[28] H. Guo, W.-x. Fu, B. Fu, K. Chen, and J. Yan, "Suboptimal trajectory programming for unmanned aerial vehicles with dynamic obstacle avoidance," Proceedings of the Institution of Mechanical Engineers, Part G: Journal of Aerospace Engineering, vol. 233, no. 10, pp. 3857-3869, 2019.

[29] R. Diestel, "Graph theory," Mathematical Gazette, vol. 173, no. 502, pp. 67-128, 2020.

[30] L. Huang, L. Wang, and C. V. Hollot, "On robust stability of polynomials and related topics," Systems Science and Mathematical Sciences, vol. 5, no. 1, pp. 42-54, 1992.

[31] Y. Cao and W. Ren, "Multi-vehicle coordination for doubleintegrator dynamics under fixed undirected/directed interaction in a sampled-data setting," International Journal of Robust and Nonlinear Control, vol. 20, no. 9, pp. 987-1000, 2010.

[32] I. Kovacs, D. S. Silver, and S. G. Williams, "Determinants of commuting-block matrices," The American Mathematical Monthly, vol. 106, no. 10, pp. 950-952, 1999. 